\title{
Modeling and Processing of Cloud Computing and It's Performance Measurement
}

\author{
Mangesh K. Nichat1, Dr. Prashant V. Ingole 2 \\ ${ }^{I}$ (Student (CSE Dept.), G H Raisoni College of Engineering and management, Amravati, India) \\ ${ }^{2}$ (Principal, G H Raisoni College of Engineering and management, Amravati, India)
}

\begin{abstract}
Cloud computing is the growing technology of large scale distributed computing. It provides ondemand access to distributed resources, which are available in open environment on paid basis. Because all most all the industries now a days want to use these services to reduce infrastructure and maintenance cost, therefore the load on cloud is increasing day by day. Balancing load is one of the biggest issue that cloud computing is facing today [1]. Many clients from all around the world are demanding the various services at rapid rate in the recent time [2]. Load balancing helps to achieve a high user satisfaction and resource utilization ratio by ensuring an efficient and fair allocation of every computing resource [3]. In the present project, a dynamic load management system has been proposed for distribution of the entire incoming request.

Keywords: Ant Colony optimization, Cloud Computing, Load balancing, , Round Robin Algorithm
\end{abstract}

\section{Introduction}

In the upcoming years the term Cloud Computing has become an emerging and fastest technology in the world. Cloud Computing is one of the most talked about technologies and due to the various opportunities offered by it has got lots of attention from media and analysts. Cloud computing is an on demand service in which shared resources, information services, software and other services are given to specific users according to the need at exact time. Cloud Computing has become one of the popular technology adopted by both industry academia providing a flexible and efficient way to store and retrieve files. The major problem is scheduling of the incoming request so minimum response time is obtained, efficient resource utilization.

The objective of this this paper is to give a brief and systematic approaches as follows.-

1. To develop a server that analyses load on different application servers in real time and redirects the user to the least loaded server for performing transactions

2. To develop an intelligent system that serves users with best performance by connecting them to least loaded and best available resources.

\section{Literature Review}

Cloud computing is rapidly gaining more attention among the researchers. In this section, the load balancing in cloud computing and its performance measurement related work is reviewed. Many researchers have done work in the field of load balancing and measured its performance by considering different parameters as discussed below.

Gulshan Soni [s] and Mala Kalra[s] proposed Central Load Balancer (CLB) technique, we tried to avoid the situation of over loading and under loading of virtual machines. The Central Load Balancer (CLB) manages load distribution among various virtual machines and assigns load corresponding to their priority and states [3].

Rajwinder Kaur and Pawan Luthra [4] stated Load balancing is helped to distribute the dynamic workload across multiple nodes to ensure that no single node is overloaded. It helps in proper utilization of resources .It also improve the performance of the system. Many existing algorithms provide load balancing and better resource utilization. Following are different types of algorithm. This is mainly divided into two categories: static load balancing algorithm and dynamic load balancing algorithm:

1) Static approach: - This approach is mainly defined in the design or implementation of the system. Static load balancing algorithms divide the traffic equivalently between all servers.

2) Dynamic approach: - This approach considered only the current state of the system during load balancing decisions. Dynamic approach is more suitable for widely distributed systems such as

\section{Cloud Computing:}

Dynamic load balancing approaches have two types .They are distributed approach and non-distributed (centralized) approach. It is defined as following:

a) Centralized Approach:- In centralized approach, only a single node is responsible for managing and distribution within the whole system. Other all nodes are not responsible for this. 
b) Distributed Approach:- In distributed approach, each node independently builds its own load vector. Vector collecting the load information of other nodes. All decisions are made locally using local load vectors. Distributed approach is more suitable for widely distributed systems such as cloud computing.

Biologists have demonstrated that several ant species are able to select cooperatively the shortest route among a set of alternative routes from their nest to a food source. In fact, each ant leaves information on the path that it has traversed by depositing a chemical substance called pheromone. The ants have tendency to follow a path rich on pheromone rather than a poorer one. Within a fixed period, shorter paths between the nest and the source of food are traversed more often than longer one. So, the quantity of pheromone is accentuated on shorter paths, which in turn augment the number of ants. This is called the reinforcement process. Inspired from this behavior of real ants, Marco Dorigo proposed the ACO metaheuristic [5] in the early 1990s to find approximate solutions to difficult optimization problems. ACO was initially applied to the travelling salesman problem (TSP), and then it has been used to solve several research problems such as the path planning problem for a mobile robot [6].

Hangoo Jeon, Young-Gi Min, Kwang-Kyu Seo[7] propose a reliable cloud service for providing objective, quantitative and measurable performance results on cloud service. In particular, this paper will define the scope for measuring the system performance of VM for IaaS among various areas of cloud service, while focusing on the area of requirements to deduce performance measurement items.

\section{Working of Cloud Computing Model:-}

\section{Methodology}

- Initially, when a registered and authorized user can login through its authentication details (username and password) for processing, their own requests is received first on the redirection server.

- Redirection server will Check the load database

- Redirection server will find most appropriate application server with least load on that application server.

- After finding least loaded server, user will connected with the least loaded server

- When user will login to cloud application server the load of that particular application server will be increased by one. Whenever after completion of user request when user logout from the cloud application server load on server will be deducted by one. In this way update the load with every user login and logout

- Performance of every server is tracked by examining the average queue time of every server's user.

- In case of emergency, when all the three application servers are overloaded up to its limit then users request are redirected towards the intermediate server for processing. The intermediate server consist of application server in emergency.

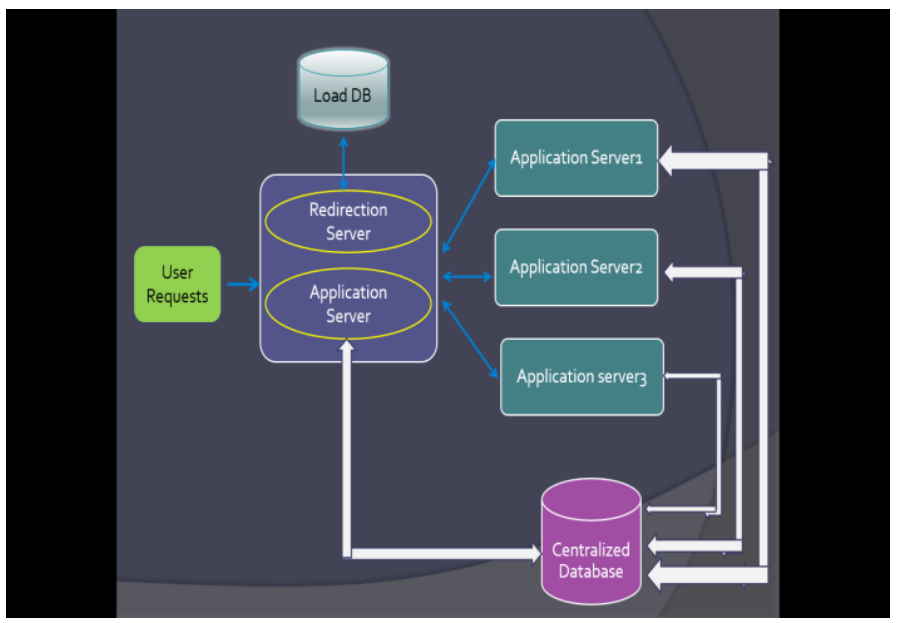

Fig. Working Model of Load Balancing in Cloud

\section{Ant Colony Optimization:}

Ants are social insects that live in colonies and whose behavior is directed more to the survival of the colony as a whole than to that of a single individual component of the colony. Social insects have captured the attention of many scientists because of the high structuration level their colonies can achieve, especially when compared to the relative simplicity of the colony's individuals. An important and interesting behavior of ant colonies is their foraging behavior, and, in particular, how ants can find shortest paths between food sources and their nest [8]. Ants are capable of finding the shortest path from food source to their nest or vice versa by smelling pheromones which are chemical substances they leave on the ground while walking. 
Each ant probabilistically prefers to follow a direction rich in pheromone. This behavior of real ants can be used to explain how they can find a shortest path [9]. The main idea is that it is indirect local communication among the individuals of a population of artificial ants.

The core of ant's behavior is the communication between the ants by means of chemical pheromone trails, which enables them to find shortest paths between their nest and food sources. This behavior of real ant colonies is exploited to solve optimization problems [10].

\begin{tabular}{|c|c|}
\hline Step 1: & $\begin{array}{l}\text { Initialize } \\
\text { Pheromone trail }\end{array}$ \\
\hline Step 2: & $\begin{array}{l}\text { Iteration } \\
\text { Repeat for each ant } \\
\text { Solution construction using pheromone trail } \\
\text { Update the pheromone trail } \\
\text { Until stopping criteria }\end{array}$ \\
\hline
\end{tabular}

\section{Round Robin Scheduling Algorithm with Example}

Round robin is the scheduling algorithm used by the CPU during execution of the process. Round robin is designed specifically for time sharing systems. It is similar to first come first serve scheduling algorithm but the preemption is the added functionality to switch between the processes.

A small unit of time also known as time slice or quantum is set/defined. The ready queue works like circular queue. All processes in this algorithm are kept in the circular queue also known as ready queue. Each New process is added to the tail of the ready/circular queue by using this algorithm, CPU makes sure, time slices (any natural number) are assigned to each process in equal portions and in circular order, dealing with all process without any priority.

It is also known as cyclic executive. The main advantage of round robin algorithm over first come first serve algorithm is that it is starvation free. Every process will be executed by CPU for fixed interval of time (which is set as time slice). So in this way no process left waiting for its turn to be executed by the CPU.Round robin algorithm is simple and easy to implement. The name round robin comes from the principle known as round robin in which every person takes equal share of something in turn.

\section{Result Analysis}

Figure shows graph for Ant Colony Optimization and Round Robin Algorithm analysis. If we observe graph it will depict that Ant Colony Algorithm will take less computation time as compared to Round robin Algorithm.

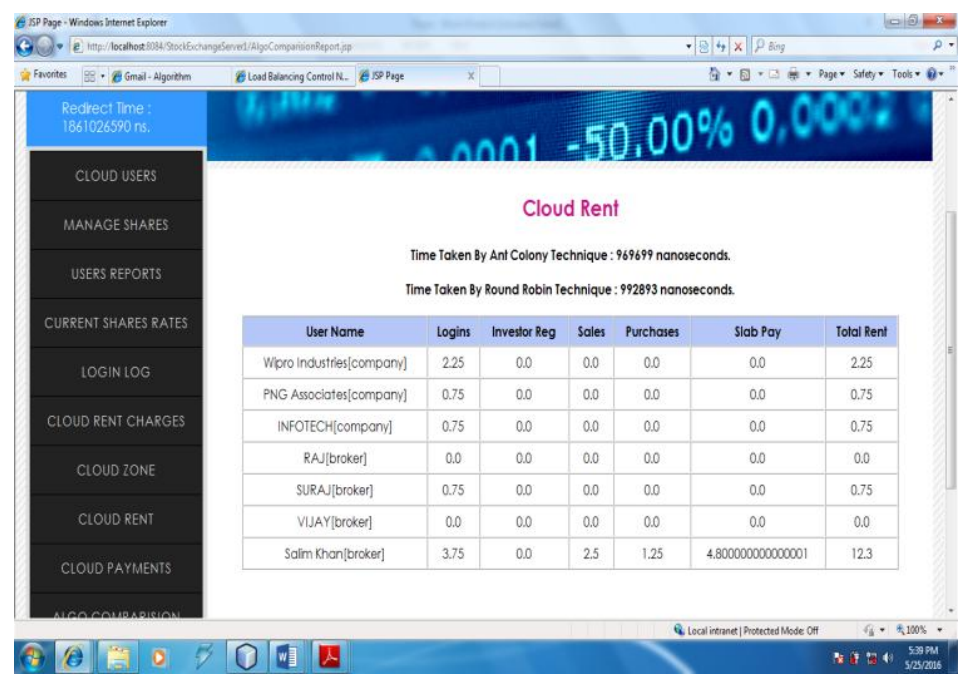




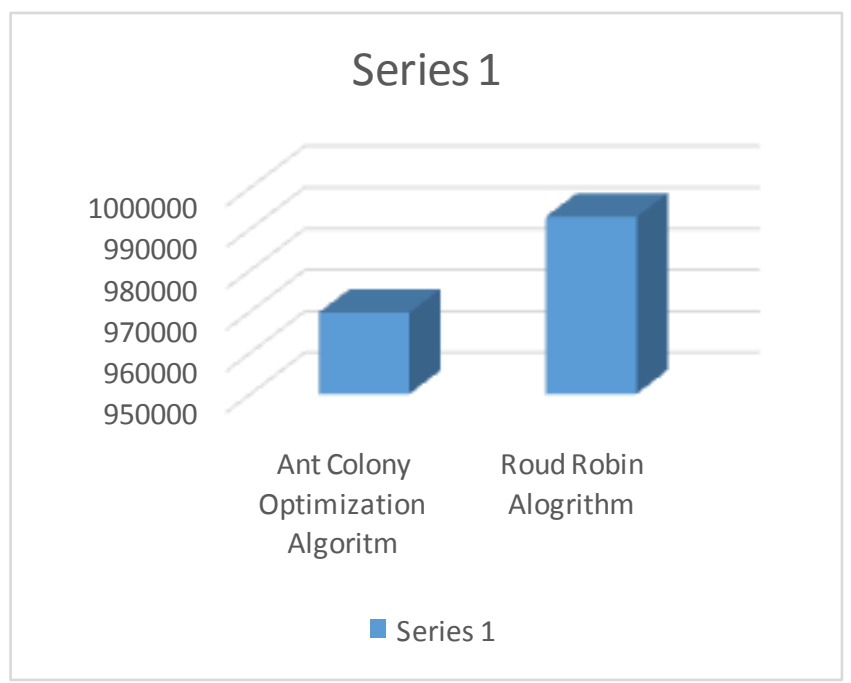

Graph shows comparison between computation time between Ant Colony Algorithm and Round Robin Algorithm in cloud.

\section{Conclusion}

With increasing popularity of large scale server side applications redirecting users to appropriate resources is very important to serve the users efficiently. This project is specifically focused on efficient load balancing so that waiting time will be reduced and improves performance of system by reducing waiting time of user request. Here, we consider some of the algorithms to measure performance of the cloud model but we can also apply other algorithms in future which are more efficient.

The unique feature of load balancer in this project is, its own application processing capability. It means control node server can perform two tasks that is redirection of users request to least loaded server and process user request when all other application servers are overloaded. So, we can conclude that this model will reduce the waiting time of user request and will do efficient resource utilization. As waiting time is reducing, performance of system will definitely increases.

\section{References}

[1]. Garima Rastogi, Dr Rama Sushil, "Analytical Literature Survey on Existing Load Balancing Schemes in Cloud Computing", International Conference on Green Computing and Internet of Things (ICGCloT), 2015.

[2]. Reena Panwar, Prof. Dr. Bhawna Mallick, "Load Balancing in Cloud Computing using Dynamic Load Management Algorithm", International Conference on Green Computing and Internet of Things (ICGCloT), 2015.

[3]. Gulshan Soni, Mala Kalra, "A Novel Approach for Load Balancing in Cloud Data Center”, IEEE International Advance Computing Conference (IACC), 2014

[4]. Rajwinder Kaur and Pawan Luthra, "Load Balancing in Cloud Computing", Association of Computer Electronics and Electrical Engineers, 2014.

[5]. Marco Dorigo, Thomas Stutzle, “Ant colony optimization.”, Cambridge, Massachusetts London, England, The MIT Press, 2004.

[6]. Imen Chaari, Anis Koubaa, Sahar Trigui, Hachemi Bennaceur, Adel Ammar and Khaled Al-Shalfan, "Smart PATH: An Efficient Hybrid ACO-GA Algorithm for Solving the Global Path Planning Problem of Mobile Robots," International Journal of Advanced Robotic Systems, (26 Mar 2014).

[7]. Hangoo Jeon, Young-Gi Min, Kwang-Kyu Seo, "A Framework of Performance Measurement of Cloud Service Infrastructure System for Service Delivery”, Advanced Science and Technology Letters Vol.46 (Cloud and Super Computing 2014), pp.142145,2014 .

[8]. Dorigo, M., Di Caro, G., \& Gambardella, L. M, “Ant Algorithms for Discrete Optimization,” Artificial Life, MIT press, 1999.

[9]. Eshghi, K., \& Kazemi, M., "Ant colony algorithm for the shortest loop design problem," Computers \& Industrial Engineering, 50, 358-366, 1999.

[10]. Baskan, O., \& Haldenbilen, S., "Ant Colony Optimization Approach for Area Traffic Control," In-Tech open Access Book, 205$220,2011$. 